\title{
EVALUATION OF MULTIPLE COMPARISON CORRECTION PROCEDURES IN DRUG ASSESSMENT STUDIES USING LORETA MAPS
}

\author{
Joan Francesc Alonso ${ }^{1,2,3}$, Sergio Romero ${ }^{1,3}$, Miguel Ángel Mañanas ${ }^{1,2,3}$, \\ Mónica Rojas ${ }^{3,1}$, Jordi Riba ${ }^{4,5,6}$, Manel José Barbanoj ${ }^{4,5,6}$ ↔
}

1. Biomedical Engineering Research Center, Department of Automatic Control, Universitat Politècnica de Catalunya. BarcelonaTech (UPC), Barcelona, Spain.

2. Barcelona College of Industrial Engineering (EUETIB), UPC, Barcelona, Spain.

3. CIBER de Bioingeniería, Biomateriales y Nanomedicina (CIBERBBN), Spain.

4. Drug Research Center, Hospital de la Santa Creu i Sant Pau, Barcelona, Spain.

5. Department of Pharmacology and Therapeutics, Universitat Autònoma de Barcelona (UAB), Barcelona, Spain.

6. CIBER de Salud Mental (CIBERSAM), Spain.

\& In memoriam - Manel J. Barbanoj passed away during the preparation of this manuscript. Rest in peace.

\begin{abstract}
The identification of the brain regions involved in the neuropharmacological action is a potential procedure for drug development. These regions are commonly determined by the voxels showing significant statistical differences after comparing placebo-induced with drugelicited effects. LORETA is an electroencephalography (EEG) source imaging technique frequently used to identify brain structures affected by the drug. The aim of the present study was to evaluate different methods for the correction of multiple comparisons in the LORETA maps. These methods which have been commonly used in neuroimaging and also simulated studies have been applied on a real case of pharmaco-EEG study where the effects of increasing benzodiazepine doses on the central nervous system measured by LORETA were investigated.. Data consisted of EEG recordings obtained from 9 volunteers who received single oral doses of alprazolam $0.25 \mathrm{mg}, 0.5 \mathrm{mg}$ and $1 \mathrm{mg}$, and placebo in a randomized cross-over double-blind design. The identification of active regions was highly dependent on the selected multiple test correction procedure. The combined criteria approach known as cluster mass was useful to reveal that increasing drug doses led to higher intensity and spread of the pharmacologicallyinduced changes in intracerebral current density.
\end{abstract}

\section{Keywords}

EEG tomography; LORETA; multiple testing; nonparametric permutation test; cluster mass; alprazolam. 


\section{INTRODUCTION}

The identification of brain regions targeted by neuropsychopharmacological agents is a newly-advanced and promising tool for drug discovery and development. Neuroimaging techniques applied to neuropharmacology provide information concerning the regional specificity of drug action, its time course, dose-response effects, and effects on cognition [17].

Localizing neural activity in the brain, both in time and in space, is an essential step to advance the understanding of cerebral function. Several non-invasive neuroimaging techniques allow the study of the cerebral functional activity: positron emission tomography (PET), single photon emission computed tomography (SPECT), functional magnetic resonance imaging (fMRI) and EEG/MEG source imaging. Some of these methods (PET, SPECT and fMRI) measure neuronal activity indirectly by quantifying glucose metabolism or cerebral blood flow [11]. The most widely used method in neuropsychopharmacology is EEG source imaging. This technique localizes the electrical brain sources responsible for the scalp potential distribution by estimating the solution to the so-called inverse problem. Scalp voltage data (EEG) is recorded after drug/placebo administration, a procedure known as pharmaco-EEG. fMRI has also been used to examine the acute effects of various drugs on neural activity [16], an approach that has been termed pharmacological MRI or phMRI. Regarding EEG- and MEG-based source imaging techniques, the EEG rhythms reflect the activation and inactivation of bidirectional connections between several cortical and subcortical structures and thus a single dipolar source indicates the centroid of the distributed cortical sources generating the EEG rhythms [21]. An alternative approach for modeling these sources is known as LORETA (low resolution electromagnetic tomography) which uses thousands of dipolar sources within a 3D brain model. LORETA takes into account anatomical constraints and composes the linear inverse solution that maximizes the synchronization among neuronal populations in terms of orientation and strength [22].

EEG/MEG source imaging is useful to study the cortical sources of EEG rhythms and evoked potentials after drug administration $[4-5,14]$. Indeed, LORETA has been used for the evaluation and characterization of the pharmacodynamics of different psychotropic drugs [28].

EEG source imaging, as every neuroimaging method, has its own strengths and weaknesses. It is a cost-effective and movement-tolerant technique and provides data with high temporal resolution but its spatial resolution is limited compared to that of phMRI. Ultimately, all the neuroimaging techniques have common issues since the brain regions involved in pharmacological action are determined by the voxels showing significant differences compared to controls or placebo. Following this methodology, an intracerebral effect map is constructed by applying a thresholding rule to the resulting statistics, and statistical maps or images are created to assess the pharmacological effect. Naive thresholding of 6239 voxels used by the LORETA approach at 5\% statistical significance is inadequate because up to 312 false positives would be expected in null data. False positives must be controlled over all tests but there is no single measure of type I error in multiple testing problems. The standard measure is the probability of any type I error, the family-wise error rate (FWE). However, other alternative procedures are constantly being developed such as the false discovery rate (FDR) error metric that inspects the expected ratio of rejected hypotheses that are false positives [8]. Several FWE testing procedures have been used in functional neuroimaging, in particular in the fMRI field [18], such as the Bonferroni approach or other methods based on the maximum statistic (minimum p-value) distribution, the random field theory, or resampling procedures [20]. However, only two approaches have been reported in the literature to control the FWE in pharmaco-EEG/MEG source imaging techniques: the nonparametric permutation test based on voxel intensity [19], and the minimum cluster size based on the binomial test [12]. The former is the multiple comparison correction procedure implemented in the LORETA statistical package and thus the most commonly used approach in pharmaco-EEG studies $[1,14,24,30]$. The performance of voxel intensity-based tests has already been examined and validated by Monte Carlo simulations of noisy cluster images with multiple intensities and diameters using the same permutation statistical tests [15]. The binomial test has also been applied in pharmaco-EEG studies, especially when drug effects are weak [3, 27, 29]. 
The main aim of this paper was to evaluate multiple comparison procedures when analyzing the effect of increasing drug doses on brain regions involved in the pharmacological action. We assessed changes in intensity, in spread of the target brain area, and in both effects combined. The drug analyzed in this study was alprazolam, which is a potent short-acting benzodiazepine. It is already known that varying a benzodiazepine dose leads to changes in the topographical EEG-based maps [6]. Thus, changes on the tomographical analysis are also expected. Three FWE methods for multiple testing were compared for the assessment of three increasing acute oral doses of alprazolam. Two of them are commonly used in pharmacoEEG/MEG neuroimaging: the nonparametric permutation test based on voxel intensity and the minimum cluster size based on the binomial test. A third procedure for FWE multiple testing was also considered: the nonparametric permutation test based on a cluster mass combining function [10]; widely used in the fMRI field. This method allows determining regions whose extent and intensity are sufficiently large, even when they are not significant by intensity or extent alone. Cluster mass performance is already known [15] but, to the best of our knowledge, has never been used before for pharmaco-EEG/MEG neuroimaging.

\section{METHODOLOGY}

\subsection{Study design}

Nine young healthy male volunteers aged between 20 and 32 years (mean age 23 years) were recruited for the study. Medical history was checked for absence of psychiatric and neurological disorders, and after medical examination, laboratory tests, ECG, and urinalysis were found to be normal. Volunteers were not allowed to take any medication for 2 weeks before the study and they abstained from alcohol, tobacco and caffeinated drinks for $48 \mathrm{~h}$ before each experimental day. Experimental days were separated by 1-week washout period.

The study was approved by the local Ethics Committee and the Spanish Ministry of Health, and was conducted in accordance with the Declaration of Helsinki and the guidelines of Good Clinical Practice. All volunteers gave their written informed consent to participate. Single oral doses of placebo and $0.25,0.5$ and $1.0 \mathrm{mg}$ of alprazolam, packaged into identical capsules, were administered in a balanced order according to a double-blind, randomized, cross-over and placebo-controlled design. Upon arrival in the laboratory under fasting conditions, a urine sample was obtained to test for illicit drug intake, electrodes were applied to the scalp and medication was given. Volunteers were seated in a comfortable reclining chair in a soundattenuated and electrically shielded chamber during each recording session, while spontaneous EEG signals with eyes closed were recorded under continuous vigilance.

The EEG recordings were acquired from 19 electrodes placed on the scalp according to the international 10/20 system on the following locations: Fp1, Fp2, F7, F3, Fz, F4, F8, T3, C3, Cz, $\mathrm{C} 4, \mathrm{~T} 4, \mathrm{~T} 5, \mathrm{P} 3, \mathrm{Pz}, \mathrm{P} 4, \mathrm{~T} 6, \mathrm{O} 1$ and $\mathrm{O} 2$, referenced to averaged mastoids. Additionally, vertical and horizontal EOG (VEOG and HEOG, respectively) signals were recorded. The VEOG was obtained from mid-forehead $(2.5 \mathrm{~cm}$ above the interpupillary line) and from the average of two electrodes below the eyes $(2.5 \mathrm{~cm}$ below the interpupillary line), whereas the HEOG signal was acquired from the outer canthi. The EEG and EOG signals were recorded at a sampling frequency of $500 \mathrm{~Hz}$ by means of Neuroscan Synamps amplifiers and analogically filtered between 0.1 and $50 \mathrm{~Hz}$. Finally, the EEG and EOG data were offline resampled with a resulting sampling frequency of $100 \mathrm{~Hz}$ and a low-pass filtering up to $40 \mathrm{~Hz}$. Three-minute EEG was recorded before drug intake (baseline) and at $+30 \mathrm{~min},+45 \mathrm{~min},+1 \mathrm{~h},+1.5 \mathrm{~h},+2.5 \mathrm{~h},+4 \mathrm{~h},+6 \mathrm{~h}$ and $+8 \mathrm{~h}$ after drug administration. To ensure the quality of the recordings, electrode impedances were checked before each acquisition and were kept below $5 \mathrm{k} \Omega$.

\subsection{Artifact minimization}

Artifacts were dealt with by using a two-step process. Its first stage involved an ocular contamination reduction process based on blind source separation (BSS). BSS-based reduction of ocular artifacts has proven to achieve more reliable tomographic maps than minimization based on regression [26]. EEG signals were segmented into 5-s epochs. A second stage consisting of an automatic artifact (saturation, muscular and movement) rejection procedure was applied as described by Anderer et al. [2]. 
BSS-based techniques decompose the multichannel EEG and EOG data into a mixture of source signals and second-order statistics are usually sufficient to solve the linear BSS problem if temporal information is taken into account. The SOBI (Second Order Blind Identification) algorithm [7], based on eigenvalue decomposition of a linear combination of several time delayed covariance matrices, was applied in this study. Finally, after the automatic detection of ocular sources, carried out using frequency and scalp topographical aspects of the source components as described by Romero et al. [25], the corrected EEG signals were reconstructed from the remaining source components.

\subsection{LORETA analysis}

Following the two-step artifact minimization, the artifact-free epochs were considered for further analysis: under the administration of placebo (30.38 \pm 6.64 epochs), alprazolam $0.25 \mathrm{mg}$ (29.03 \pm 8.67 epochs), alprazolam $0.5 \mathrm{mg}$ (32.05 \pm 4.71 epochs), and alprazolam $1 \mathrm{mg}$ (27.93 \pm 8.03 epochs). The LORETA technique was used to estimate the three-dimensional intracerebral current density function from the voltage values recorded at the scalp electrodes. Specifically, the sLORETA (standardized low resolution brain electromagnetic tomography) version of the program was employed in this study, as it provides the lowest possible localization error when testing point sources [22]. The 3D localization maps were obtained by calculating a locationwise inverse weighting of the minimum-norm least-squares inverse solution with estimated variances. This EEG source imaging technique restricts the solution to 6239 cortical grey matter voxels with a resolution of $5 \mathrm{~mm}$ (spatial resolution of $0.125 \mathrm{~cm}^{3}$ ) according to the digitized MNI (Montreal Neurological Institute) Human Brain Atlas [9].

In a first step, the current density values were estimated based on the EEG cross-spectral matrix and then squared for each voxel in the following frequency bands: delta ( 0.5 to $3.5 \mathrm{~Hz})$, theta (3.5 to $7.5 \mathrm{~Hz})$, alpha1 $(7.5$ to $10.5 \mathrm{~Hz})$, alpha2 (10.5 to $13 \mathrm{~Hz})$, beta1 (13 to $16 \mathrm{~Hz})$, beta2 (16 to $20 \mathrm{~Hz}$ ), beta3 (20 to $25 \mathrm{~Hz})$, beta4 $(25$ to $30 \mathrm{~Hz}$ ), and beta5 (30 to $35 \mathrm{~Hz}$ ). To evaluate differences between drugs and placebo, paired-sample t-tests were computed for the baselinecorrected (subtraction of the baseline values) and log-transformed LORETA power values in each voxel and for each frequency band at the different time points. The large number of statistical tests that were carried out simultaneously due to the number of voxel-wise comparisons resulted in an increased risk of type I errors. To control for this increased risk of false positives, the three multiple comparison correction methods mentioned above were applied to the data to identify the number of significant voxels in each region of interest. As explained in the introduction, the first two methods have been commonly used in pharmaco-EEG neuroimaging, whereas the latter comes from the fMRI field and has not been applied before to this neuroimaging approach.

\subsubsection{Nonparametric permutation test based on voxel intensity or peak height}

The nonparametric permutation test [19] is a single threshold test based on the theory of randomization tests. In particular, the voxel intensity procedure calculates the critical t-value using a random sample of all the possible permutations and estimates the distribution of the maximum $t$ statistic. Under the null hypothesis, data labels can be permuted without altering the distribution of the test statistic of interest. Corrected p-values or threshold statistical voxel intensities can be calculated from this empirical distribution. This distribution depends on the correlation structure of the t-statistic, and is considered a useful method to set the critical tthreshold.

\subsubsection{Minimum cluster size using the binomial test}

A single null hypothesis for omnibus significance based on the binomial test was also employed. This method considers the size of the target region and calculates the minimum number of contiguous voxels constituting a suprathreshold region by means of a binomial test [12]. For the 6239 voxels obtained by LORETA and an overall significance of 5\%, a voxel could be considered significant if it belonged to a cluster with at least 341 voxels according to the binomial theorem. Clusters were built as a set of contiguous voxels whose t-value exceeded the corresponding threshold ( $\mathrm{t}=2.306$ in this study, corresponding to a significance of 0.05 and 8 degrees of freedom) considering an 18-connectivity scheme, that is, neighbors sharing at least 
one edge with the considered voxels.. Hence, the active region derived from this method has to be large and compact enough to be considered a consequence of the drug effects and not of mere statistical chance.

\subsubsection{Nonparametric permutation test based on cluster mass combining function}

A combined statistic of voxel intensity and cluster size can be used to benefit from the strength of both FWE measures. Thus, the cluster mass combining function can produce significant results when both intensity and cluster size are marginally significant $[10,15]$. In particular, the cluster mass is calculated by adding up the intensity values exceeding the t-value threshold of all the voxels in a cluster. This summation is computed over all the clusters and considering all the possible permutations, so that an empirical distribution of the largest cluster mass values obtained can be used to find FWE-corrected p-values for the clusters derived from the original labeling.

a)
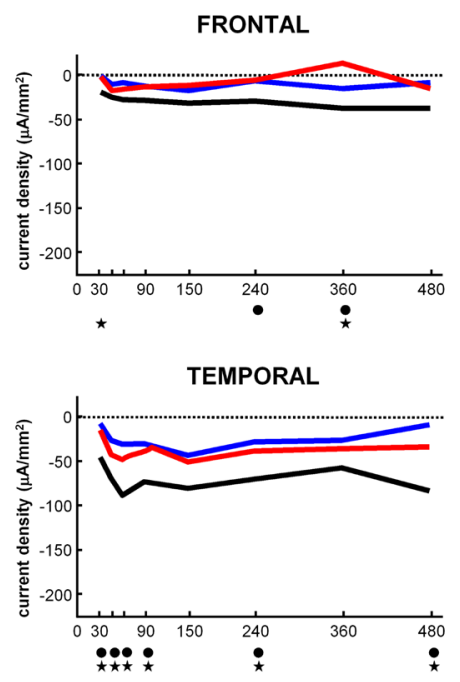

b)

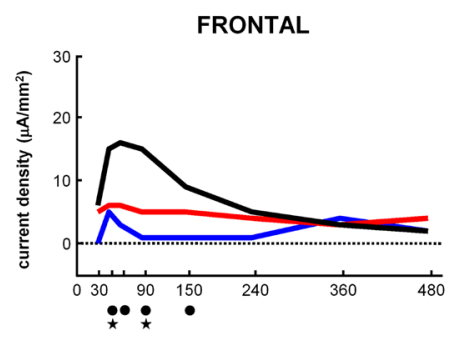

TEMPORAL

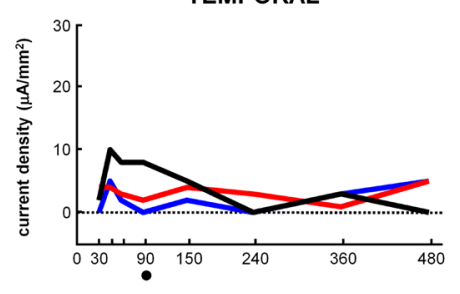

ALPHA1

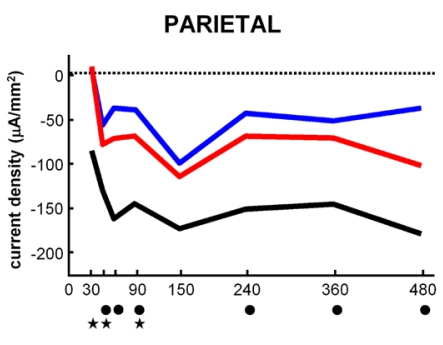

LIMBIC

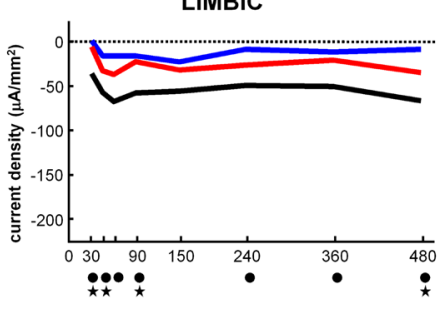

BETA1

PARIETAL

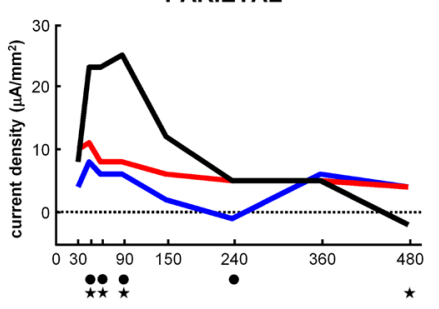

LIMBIC

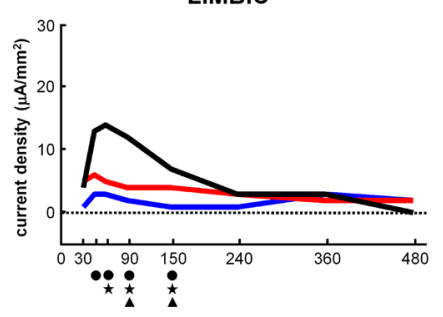

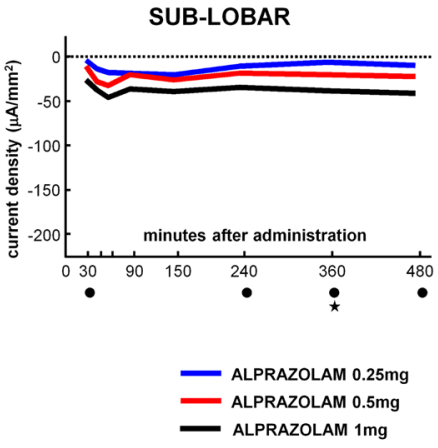

- ALPRAZOLAM $0.25 \mathrm{mg}$

ALPRAZOLAM $0.5 \mathrm{mg}$

OCCIPITAL

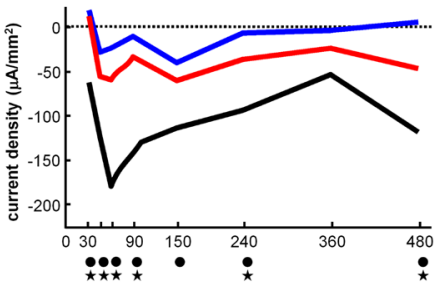

OCCIPITAL

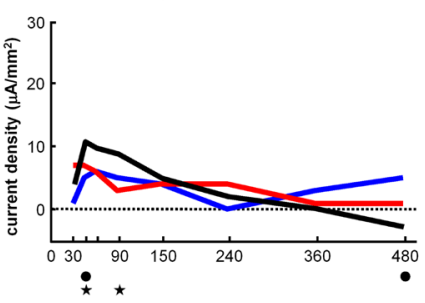

SUB-LOBAR

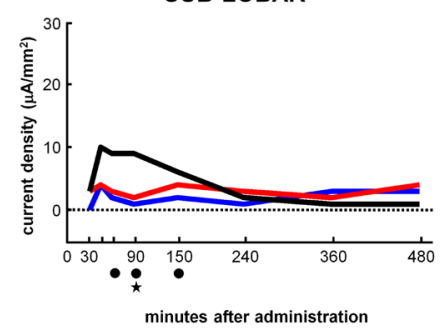

Fig. 1: Time course of average current source density values $\left(\mu \mathrm{A} / \mathrm{mm}^{2}\right)$ obtained in the different brain lobes after baseline and placebo subtraction for the a) alpha1 and b) beta1 bands. Different y-axis scales were used for the alpha1 and betal graphs. The doses of alprazolam are depicted in different shades of grey. Statistical comparisons between doses of alprazolam are depicted using the following symbols: circle, $1 \mathrm{mg} v s 0.25 \mathrm{mg}$; asterisk, $1 \mathrm{mg} v s \quad 0.5 \mathrm{mg}$; and triangle, $0.5 \mathrm{mg} v s 0.25 \mathrm{mg}$. 


\section{RESULTS}

The sedative-hypnotic effect of alprazolam is characterized by: 1) a specific decrease in the absolute power of the slow alpha activity (alpha1), whereas the fast alpha activity (alpha2) remains unchanged; and 2) a generalized increase of the absolute power of beta activity [28].

Figure 1 shows the time course of the LORETA net current source density corresponding to the different doses of alprazolam after subtracting baseline and placebo values for the alphal and betal bands. These results indicate that alprazolam-induced effects were stronger in the alphal than in the betal band. The alphal decreases were emphasized in the parietal and occipital lobes, whereas the betal increases were accentuated in the frontal, parietal and limbic lobes. Ascending-dose effects were observed in all brain lobes for both frequency bands. However, depending on inter-subject variability of the LORETA current density values, small changes associated with low variance led to significant activations whereas large differences with perceptible variance were not significant. Statistical tests between the LORETA values corresponding to the placebo and alprazolam doses were performed at each voxel and recording time for the two frequency bands (alphal and betal) which are primarily affected by alprazolam.

Note that the acquisition of EEG signals at different times after alprazolam administration allowed the assessment of the time course of target brain regions associated with significant changes in intracerebral current density. Moreover, this time monitoring allowed the identification of the onset and the duration of the pharmacological effect in the alphal and betal frequency bands.

Three-dimensional statistical maps were obtained to test for differences in tomography between the binomial and voxel intensity multiple comparison correction methods. These maps showed the target regions at different times after the administration of the three alprazolam doses $(0.25 \mathrm{mg}, 0.5 \mathrm{mg}$ and $1 \mathrm{mg}$ in figures 2,3 and 4, respectively) compared with placebo, for the alpha1 and betal rhythms. Finally, a color gradient was used to show the statistically significant regions after applying the abovementioned correction methods. 


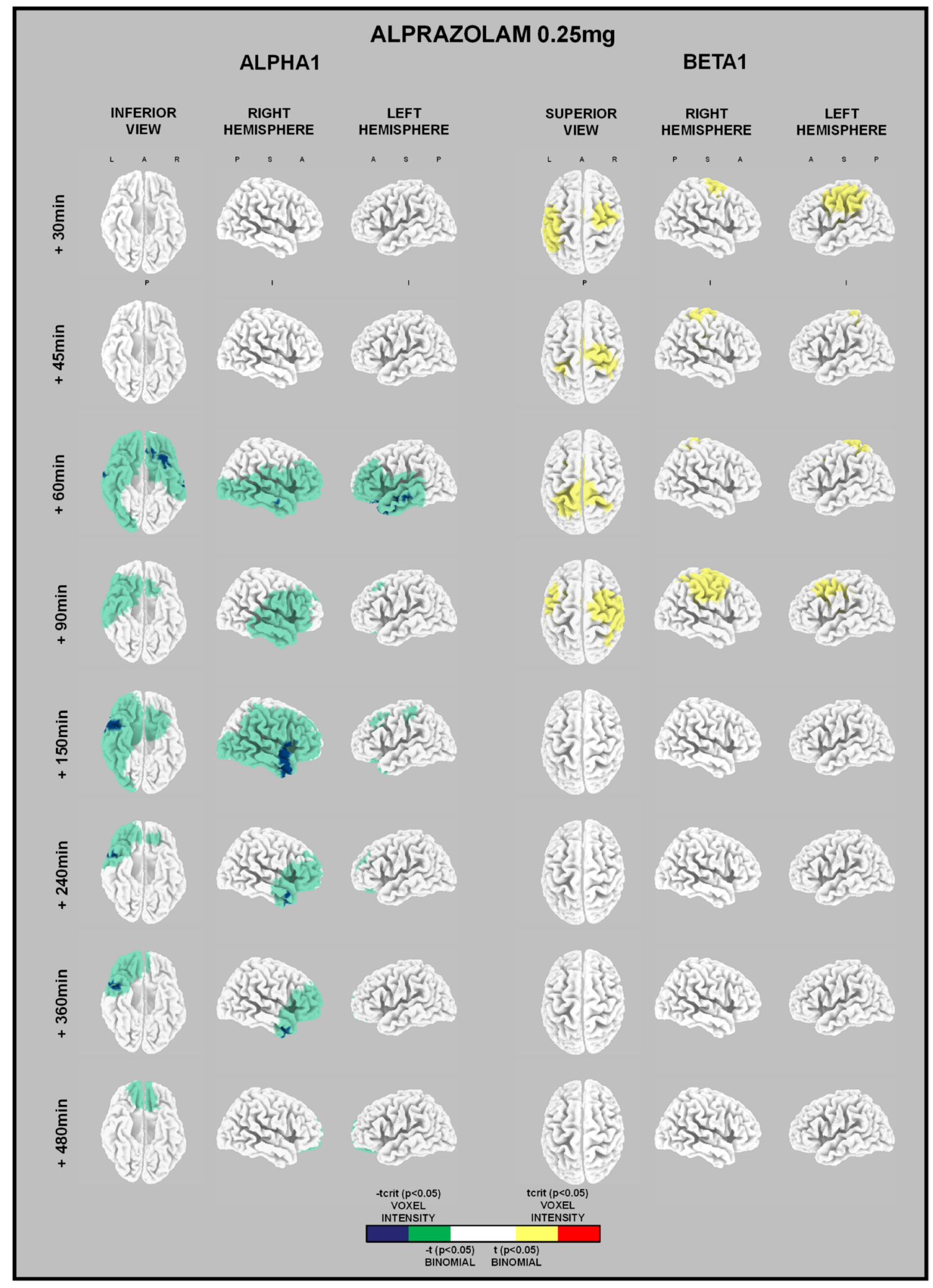

Fig. 2: Time course of the intracerebral effects after $0.25 \mathrm{mg}$ alprazolam compared with placebo $(\mathrm{n}=9)$. The images, visualized from different perspectives, show statistical parametric maps based on $t$ values of the differences between alprazolam- and placebo-induced changes with respect to baseline. Different shades of color were used to clarifv the 


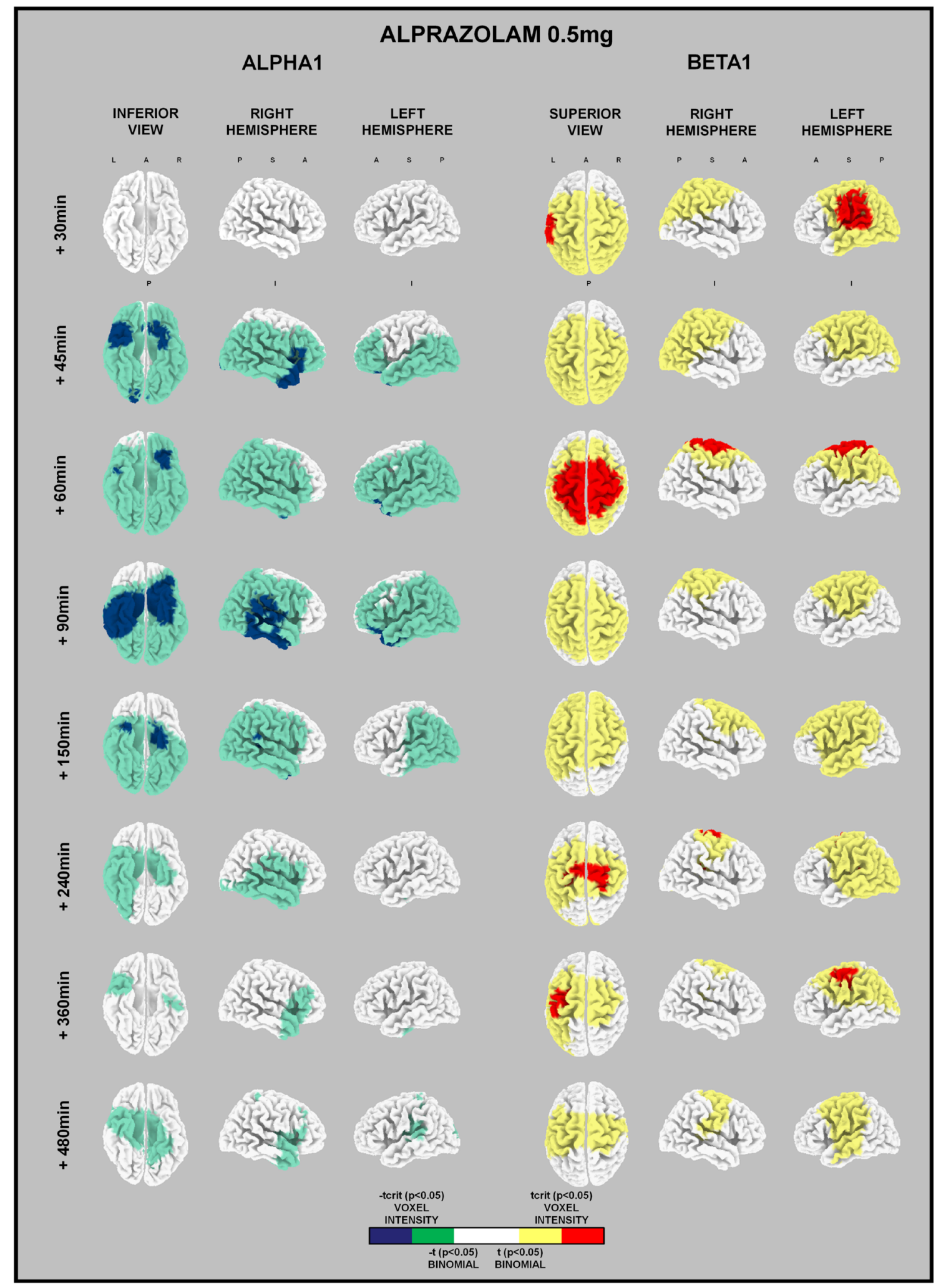

Fig. 3: Time course of intracerebral effects after $0.5 \mathrm{mg}$ alprazolam compared with placebo $(\mathrm{n}=9)$. See figure 2 legend for abbreviations and more information. 


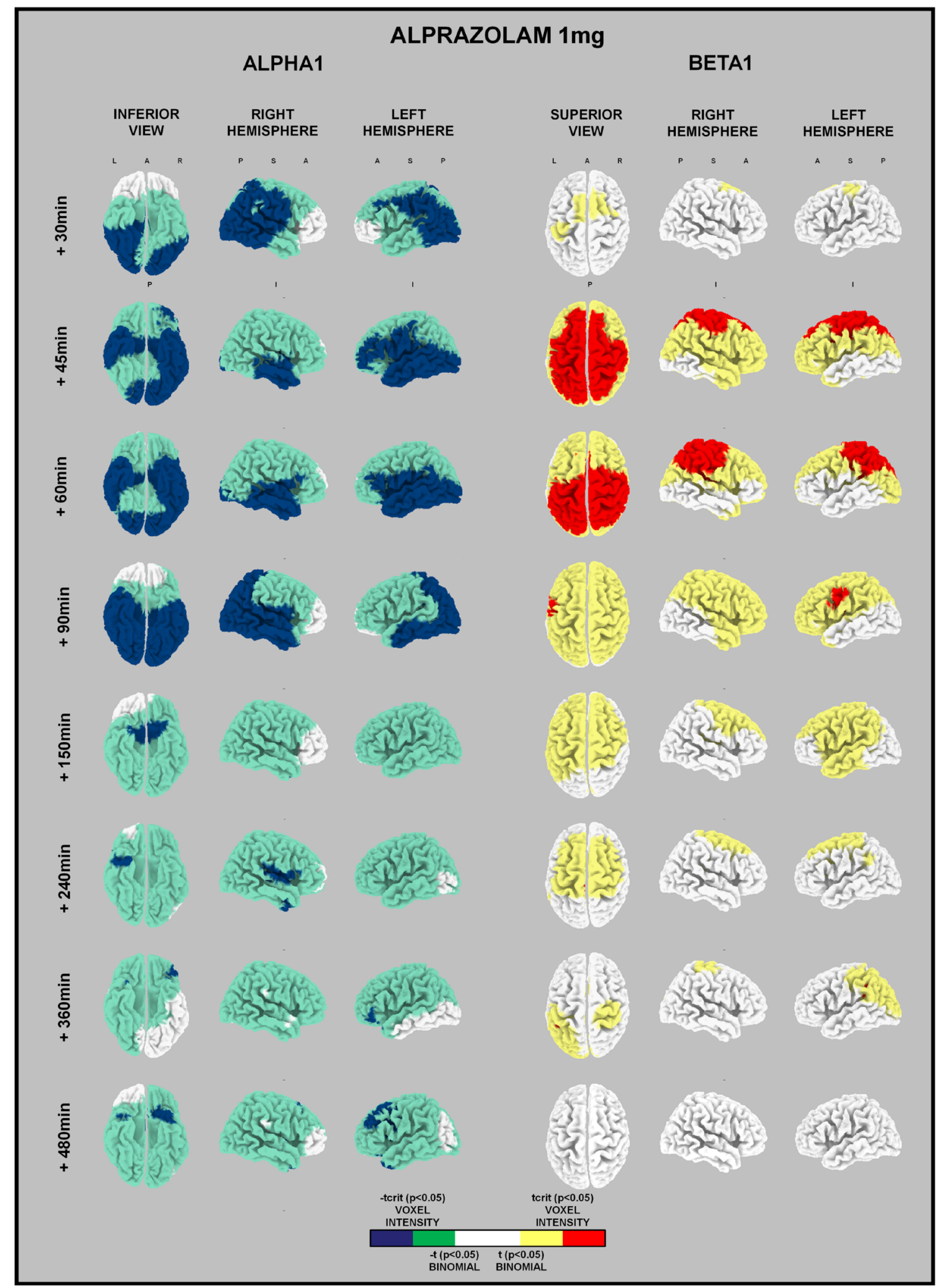

Fig. 4: Time course of intracerebral effects after $1 \mathrm{mg}$ alprazolam compared with placebo $(\mathrm{n}=9)$. See figure 2 legend for abbreviations and more information.

\subsection{Nonparametric permutation test based on voxel intensity}

The multiple comparison correction based on the voxel intensity procedure was performed using all the 512 possible randomizations [19]. The analysis at the time point when the maximum effect was expected (around 60 and 90min after drug administration) showed little or no significant differences (decreases in dark blue and increases in dark red) for the $0.25 \mathrm{mg}$ dose, neither in alpha1 nor in beta1. On the other hand, $1 \mathrm{mg}$ of alprazolam caused a significant 
decrease of alphal activity located mainly in the temporal and occipital lobes. Regarding the effects in the betal band, a significant increase in intracerebral power density was observed after the $0.5 \mathrm{mg}$ dose. Although it was located between the frontal and parietal lobes, this increase shifted towards the parietal lobes after the $1 \mathrm{mg}$ dose.

Regarding the effect duration, the effects of the $1 \mathrm{mg}$ dose appeared in alpha1 30 minutes after intake, dropped at 150 minutes, and a small action still remained even at 8 hours after administration. In contrast, the $1 \mathrm{mg}$ effect in betal was shorter: it arose at 45 minutes and vanished at 90 minutes. Nevertheless, at the low and medium alprazolam doses, the time course of drug-induced changes was not coherent with the known pharmacological effect. For instance, no significant effect was obtained for beta1 at any time after the lowest dose administration. Moreover, a very small significant change was observed in alphal after administration of the low dose. This effect appeared in the right hemisphere at 60 minutes, disappeared at 90 minutes, but then reappeared in the left hemisphere at 150 minutes. Similar results were obtained for alpha1 and especially beta1 following the medium dose administration: the effect appeared, then vanished, and reappeared later, several hours after drug administration.

\subsection{Minimum cluster size using the binomial test}

For the binomial test method, voxels were colored within a contiguous region composed of more than 341 voxels with t-values exceeding a threshold of 2.306 (corresponding to a significance level of 0.05). The results in figures 2,3 and 4 show how the intracerebral target regions (drawn in any color) expanded when the threshold t-value decreased, for both alphal and beta1 bands. In other words, when the threshold t-value is less restrictive, more voxels accomplished the condition resulting in larger significant areas. In addition, the correspondence between the ascending dose effect and the increasingly larger significant brain regions was clearly more marked for the binomial test than for the voxel intensity method, and even the 0.25 $\mathrm{mg}$ dose showed a significant region in the former case.

With respect to the figures, the target brain regions indicated a more logical temporal progression: for example, the effect induced in alphal by the medium dose did not show an intermittent behavior at consecutive time points as happened with the voxel intensity procedure. However, the binomial test method is considered a weak control of the FWE. Although it is useful for detecting whether there is any pharmacological effect, it is not suitable for the localization of the target brain regions.

\subsection{Nonparametric permutation test based on cluster mass combining function}

Table 1 shows the results of the cluster mass combining function after applying this test for the alphal and betal bands. Significant suprathreshold clusters were found by this combined test at practically all time points after the intake of the medium and high alprazolam doses compared with placebo. At the remaining $0.25 \mathrm{mg}$ dose, significant maps were obtained for alpha1 between 60 and 150 minutes.

TABLE 1: Cluster mass function and p-values of alprazolam- versus placebo-induced changes in the alpha1 and beta1 powers for three different doses. Several time points after drug administration were considered. Cluster-based statistics were found setting a threshold corresponding to a significance level of 0.05 to define the cluster area.

\begin{tabular}{|c|c|c|c|c|c|c|c|}
\hline & \multirow[b]{2}{*}{ TIME } & \multicolumn{2}{|c|}{$\begin{array}{c}\text { ALPRAZOLAM } \\
0.25 \mathrm{mg} \\
\end{array}$} & \multicolumn{2}{|c|}{$\begin{array}{c}\text { ALPRAZOLAM } \\
0.5 \mathrm{mg} \\
\end{array}$} & \multicolumn{2}{|c|}{$\begin{array}{c}\text { ALPRAZOLAM } \\
1 \mathrm{mg} \\
\end{array}$} \\
\hline & & $\begin{array}{c}\text { Cluster } \\
\text { mass }\end{array}$ & $\begin{array}{c}\text { p-value } \\
\text { cluster } \\
\text { mass } \\
\end{array}$ & $\begin{array}{l}\text { Cluster } \\
\text { mass }\end{array}$ & $\begin{array}{c}\text { p-value } \\
\text { cluster } \\
\text { mass } \\
\end{array}$ & $\begin{array}{c}\text { Cluster } \\
\text { mass }\end{array}$ & $\begin{array}{c}\text { p-value } \\
\text { cluster } \\
\text { mass } \\
\end{array}$ \\
\hline \multirow{8}{*}{ 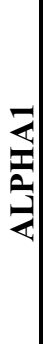 } & 30' & 260 & 0.096 & 0 & 0.976 & 12528 & 0.002 \\
\hline & 45' & 104 & 0.217 & 5466 & 0.006 & 13584 & 0.002 \\
\hline & 60 ' & 2717 & 0.002 & 6257 & 0.010 & 14884 & 0.002 \\
\hline & 90' & 1292 & 0.012 & 12767 & 0.002 & 25801 & 0.002 \\
\hline & 150' & 2203 & 0.025 & 4880 & 0.002 & 10107 & 0.002 \\
\hline & 240 ' & 795 & 0.072 & 1014 & 0.022 & 8657 & 0.006 \\
\hline & 360 & 577 & 0.057 & 328 & 0.057 & 9884 & 0.002 \\
\hline & 480 & 204 & 0.205 & 561 & 0.072 & 10511 & 0.002 \\
\hline
\end{tabular}




\begin{tabular}{c|c|cc|cc|cc}
\hline $\mathbf{3 0}$ & 224 & 0.225 & 7704 & 0.006 & 312 & 0.205 \\
$\mathbf{4 5}$, & 134 & 0.154 & 4809 & 0.006 & 10205 & 0.002 \\
$\mathbf{6 0}$, & 297 & 0.131 & 8749 & 0.002 & 13286 & 0.002 \\
$\mathbf{9 0}$ & 447 & 0.082 & 2974 & 0.020 & 7640 & 0.012 \\
$\mathbf{9 0}$ & 0 & 0.984 & 1806 & 0.029 & 3220 & 0.006 \\
$\mathbf{1 5 0}$, & 1 & 0.705 & 2734 & 0.006 & 1872 & 0.010 \\
$\mathbf{2 4 0}$, & 37 & 0.369 & 2504 & 0.014 & 1116 & 0.029 \\
$\mathbf{3 6 0}$, & 0 & 0.941 & 1013 & 0.049 & 217 & 0.150 \\
\hline
\end{tabular}

TABLE 2: Nonparametric permutation tests (partial voxel intensity and combined cluster mass) applied in order to show the statistical changes for alpha1 and beta1 powers at $90 \mathrm{~min}$ and $60 \mathrm{~min}$ after administration respectively, in the brain regions involved in the pharmacological action after an increase of the alprazolam dosage. Statistics and cluster values were found setting a threshold corresponding to a significance level of 0.05 to define the cluster area.

\begin{tabular}{|c|c|c|c|c|}
\hline \multirow{3}{*}{$\begin{array}{l}\text { COMPARED } \\
\text { TREATMENTS }\end{array}$} & \multicolumn{4}{|c|}{ ALPHA1 (90min after administration) } \\
\hline & \multicolumn{2}{|c|}{ voxel intensity } & \multicolumn{2}{|c|}{ cluster mass } \\
\hline & $\begin{array}{l}\text { Extreme } \\
\text { t-value }\end{array}$ & p-value & $\begin{array}{l}\text { Cluster } \\
\text { mass value }\end{array}$ & p-value \\
\hline $0.25 \mathrm{mg} v s$ placebo & -6.232 & 0.043 & 1292 & 0.012 \\
\hline $0.5 \mathrm{mg} v s$ placebo & -13.610 & 0.002 & 12767 & 0.002 \\
\hline \multirow[t]{2}{*}{$1 \mathrm{mg} v s$ placebo } & -16.538 & 0.002 & 25801 & 0.002 \\
\hline & \multicolumn{4}{|c|}{ BETA1 (60min after administration) } \\
\hline \multirow{2}{*}{$\begin{array}{l}\text { COMPARED } \\
\text { TREATMENTS }\end{array}$} & \multicolumn{2}{|c|}{ voxel intensity } & \multicolumn{2}{|c|}{ cluster mass } \\
\hline & $\begin{array}{l}\text { Extreme } \\
\text { t-value }\end{array}$ & p-value & $\begin{array}{l}\text { Cluster } \\
\text { mass value }\end{array}$ & p-value \\
\hline $0.25 \mathrm{mg} v s$ placebo & 4.053 & 0.107 & 297 & 0.131 \\
\hline $0.5 \mathrm{mg}$ vs placebo & 12.170 & 0.002 & 8749 & 0.002 \\
\hline $1 \mathrm{mg} v s$ placebo & 15.119 & 0.002 & 13286 & 0.002 \\
\hline
\end{tabular}

The suprathreshold clusters detected by the cluster mass method corresponded to target brain regions where peak intensity was significant. Cluster mass showed clearly the effect of ascending doses as a function of time: higher values were obtained from 45 or 60 minutes until 90 or 150 minutes after the administration for any dose, and these values increased with dose (see table 1). Table 2 shows the results of partial voxel-intensity and combined nonparametric permutation tests for alphal and betal powers after alprazolam administration, at 90 and 60 minutes respectively, compared with placebo. Note that the extreme t-values increased with the dose and the corrected p-values produced by the cluster mass test showed that significant clusters were found for all the comparisons except for betal in $0.25 \mathrm{mg} v \mathrm{~s}$ placebo. These results indicated that the effect of an ascending-dose of alprazolam produced both an increase of the intensity and an enlargement of the brain regions involved in the drug action.

According to these results, it could be concluded that the LORETA statistical probability maps and the brain regions considered suprathreshold after drug administration depended heavily on the statistical significance level. Likewise, the minimum voxel intensity, the minimum cluster mass, and their corrected p-values were conditioned by the selected significance level. Figure 5 shows the cluster mass value (integral of suprathreshold intensities within a cluster) as a function of different significance levels (from 0.05 to 0.001 ) for alpha1 and beta 1 powers, and evidences how cluster mass values decreased monotonically with lower values of significance level. A similar effect, associated with an ascending-dose of the drug, was found for all the significance levels: an increase of the alprazolam dosage entailed both a rise in the intensity and a spread of the drug-targeted areas. 
ALPHA1

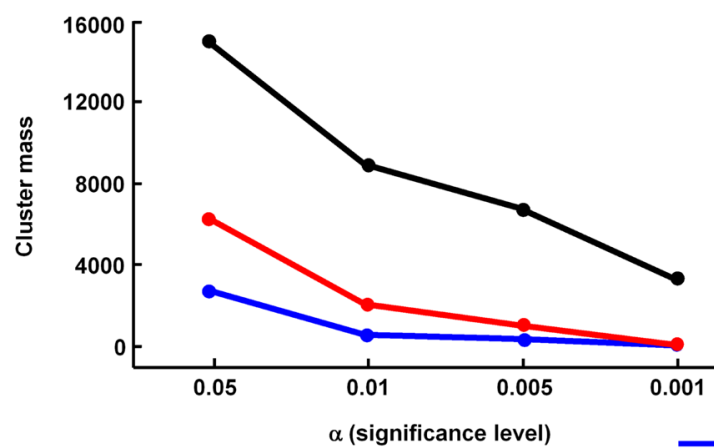

BETA1

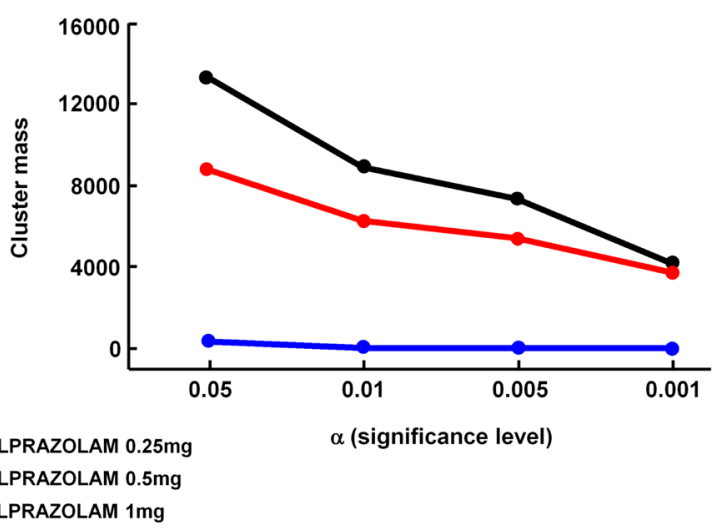

Fig. 5: Cluster mass calculated as a function of different values of significance level ( ) in alpha1 and beta1 powers at 1 hour after the administration of different doses of alprazolam compared with placebo.

\section{DISCUSSION}

The identification of the brain structures involved in pharmacological action is a promising tool to investigate and develop new drugs. The usual procedure used to identify target voxels in pharmaco-neuroimaging is to perform voxelwise hypothesis tests (between drug and placebo conditions) and to threshold the resulting statistical image. However, the literature reports several different approaches concerning the thresholding of the obtained statistical tomographic maps. Whereas conservative methods which allow for a fairly accurate localization of the target area have been employed $[14,24]$, other less conservative techniques have been used to show weaker drug effects $[3,29]$. This duality is accentuated when spontaneous EEG is used because, unlike evoked potentials, the location of the cerebral generators is less clear for spontaneous EEG and the interpretation of results is much more open and less spatially restricted.

One of the most common conservative methods is the Bonferroni correction, where each voxel is tested at a significance level divided by the total number of voxels (comparisons). This correction would increase the t-value threshold from 2.306 to 10.077 ( 1 vs. 6239 voxel comparisons), and consequently the activity level associated with pharmacological effect would have to be extremely strong to exceed the adjusted threshold. One usual way to overcome this issue is the a priori definition of regions of interest (ROI) where the drug effect is expected, however such definition is feasible for evoked potentials but not when dealing with spontaneous activity.

In EEG/MEG-based neuropsychopharmacology only the voxel intensity and the binomialbased cluster size tests have been used to date. Nonparametric permutation tests are based on setting the critical threshold according to the empirical null distribution of the test statistic of interest. This is ascertained directly from the observed data, although its calculation requires a considerable computational effort. It is important to note that, although each voxel is tested independently of its neighbors, important drug-induced differences are expected in the spatially contiguous voxels, and the nonparametric permutation method based on cluster mass takes this information into account to look for compact regions of voxels where the effect is significant. Alternatively, a simple method like the binomial test can be applied to obtain the minimum number of contiguous voxels that have to exceed the threshold to be considered significant. However, the binomial test is a weak control over the FWE and can only indicate the presence of activation. In spite of that, these weak FWE control few methods, commonly named omnibus tests, are useful for detecting the existence of any pharmacological effect regardless of location of these effects [23].

Several works have evaluated different multiple comparison procedures in fMRI analysis $[13,18,20]$. In general, multiple comparison correction procedures can be classified into 
parametric (based on random field theory) and nonparametric statistics. Parametric statistics are more robust but require a Gaussian distribution, whereas nonparametric statistics have the advantage of being distribution-independent as well as insensitive to extreme values or outliers, at an additional computational cost.

Among the evaluated correction procedures, the conservative approach of the nonparametric permutation test based on voxel intensity allowed the determination of the targeted brain region after alprazolam administration as compared with placebo. The alpha1 LORETA power decrease was mainly located in the temporal lobe, and the beta1 LORETA power increased in the parietal lobe. However, the brain regions involved did not show any coherent time or ascending-dose course. On the one hand no differences were obtained for 0.25 $\mathrm{mg}$ neither in alphal nor in beta1; on the other, significant effects usually appeared and disappeared intermittently at consecutive times, reappearing when the drug effect was theoretically very weak.

According to these results, the interpretation of the statistical tests can be determined by subjectively adjusting the thresholds (using a more or less conservative multiple comparison correction procedure) to obtain desirable results. In this sense, better results were obtained using the voxel intensity approach when the drug effect was substantial (1 $\mathrm{mg}$ dose) in contrast to the results obtained when the effect was weaker ( 0.25 and $0.5 \mathrm{mg}$ doses $)$.

The aim of this paper was to investigate if the pharmacological effects on the brain after an ascending dose were larger in extent, intensity or both. The cluster mass, calculated as the integral of suprathreshold intensities within a cluster, is a theoretical test statistic which combines spatial extent and peak height intensity [15]. It is worth noting that temporal and ascending-dose effects were clearly revealed by the cluster mass statistic (see table 2). These results were found for different significance levels, indicating that although statistics and cluster-based parameters depended on the significance level, the conclusion was robust: an increase in alprazolam dose occasioned both a rise in the intensity and a spread of the brain regions involved in the pharmacological action.

Finally, the nonparametric methods analyzed in this study were found to be appropriate and valuable tools to objectively assess the impact dose on the target brain regions. Cluster mass provided very useful information on the pharmacological effect with ascending doses. As a matter of fact, cluster mass could be considered a complementary measure to the voxel intensity and cluster size tests. In this way, the cluster mass value associated with both intensity and brain area was coherent with dose level and theoretical time intervals at which maximum effects are expected.

\section{ACKNOWLEDGEMENTS}

This study was supported in part by the Ministerio de Economía y Competividad, under contract DPI2011-22680 and DPI2014-59049R. CIBER-BBN and CIBERSAM are an initiative of the Instituto de Salud Carlos III, Spain. The authors thank the staff at the Centre d'Investigació de Medicaments (CIM) of the Biomedical Research Institute Sant Pau for their technical assistance during data collection. The present research complies with the Spanish Law.

Manel J. Barbanoj started this study and passed away during the early preparation of this manuscript. Humble, accessible, cheerful and enthusiastic of life, Manel was loved and respected by his friends and colleagues. For all this and for their important contributions in the fields of neuropsychopharmacology and sleep, he will be always present in our memories. Rest in peace. 


\section{REFERENCES}

[1] H.A. Alhaj, A.E. Massey, R.H. McAllister-Williams. Effects of DHEA administration on episodic memory, cortisol and mood in healthy young men: a double-blind, placebocontrolled study. Psychopharmacology 188, 541-551 (2006).

[2] P. Anderer, H.V. Semlitsch, B. Saletu, M.J. Barbanoj. Artifact processing in topographic mapping of electroencephalographic activity in neuropsychopharmacology. Psychiatry Res 45, 79-93 (1992).

[3] P. Anderer, B. Saletu, R.D. Pascual-Marqui. Effect of the 5-HT(1A) partial agonist buspirone on regional brain electrical activity in man: a functional neuroimaging study using low-resolution electromagnetic tomography (LORETA). Psychiatry Res 100, 81-96 (2000).

[4] P. Anderer, B. Saletu, M. Wolzt, S. Culic, A. Assandri, F. Nannipieri, et al. Double-blind, placebo-controlled, multiple-ascending-dose study on the effects of ABIO-08/01, a novel anxiolytic drug, on perception and cognition, utilizing event-related potential mapping and low-resolution brain electromagnetic tomography. Hum Psychopharmacol Clin Exp 23, 234-254 (2008).

[5] C. Babiloni, F. Frisoni, M. Steriade, L. Bresciani, G. Binetti, C. Del Percio, et al. Frontal white matter volume and delta EEG sources negatively correlate in awake subjects with mild cognitive impairment and Alzheimer's disease. Clin Neurophysiol 117, 1113-1129 (2006).

[6] M.J. Barbanoj, P. Anderer, R.M. Antonijoan, J. Torrent, B. Saletu, F. Jané. Topographic pharmaco-EEG mapping of increasing doses of buspirone and its comparison with diazepam. Hum Psychopharmacol 9, 101-109 (1994).

[7] A. Belouchrani, K. Abed-Meraim, J.F. Cardoso, E. Moulines. A blind source separation technique using second-order statistics. IEEE Trans Signal Process 45, 434-444 (1997).

[8] Y. Benjamini, Y. Hochberg. Controlling the false discovery rate: a practical and powerful approach to multiple testing. J R Stat Soc Ser B 57, 289-300 (1995).

[9] M. Brett, IS. Johnsrude, AM. Owen. The problem of functional localization in the human brain. Nature Reviews Neuroscience 3, 243-249 (2002).

[10] E.T. Bullmore, J. Suckling, S. Overmeyer, S. Rabe-Hesketh, E. Taylor, M.J. Brammer. Global, voxel, cluster tests, by theory and permutation, for a difference between two groups of structural MR images of the brain. IEEE Trans Med Imaging 18, 32-42 (1999).

[11] G. Buzsáki. Rhythms of the brain. New York: Oxford University Press (2006).

[12] E.M. Cross, W.W. Chaffin. Use of the binomial theorem in interpreting results of multiple tests of significance. Educ Psychol Meas 42, 25-34 (1982).

[13] C.R. Genovese, N.A. Lazar, T. Nichols. Thresholding of statistical maps in functional neuroimaging using the false discovery rate. Neuroimage 15, 870-878 (2002).

[14] L.R. Gianotti, G. Künig, P.L. Faber, D. Lehmann, R.D. Pascual-Marqui, K. Kochi, et al. Rivagstimine effects on EEG spectra and three-dimensional LORETA functional imaging in Alzheimer's disease. Psychopharmacology 198, 323-332 (2008).

[15] S. Hayasaka, T. Nichols. Combining voxel intensity and cluster extent with permutation test framework. Neuroimage 23, 54-63 (2004).

[16] G. Honey, E. Bullmore. Human pharmacological MRI. Trend Pharmacol Sci 25, 366-374 (2004).

[17] M.D. Ireland, S.C.R. Williams. Application of pharmacological MRI to preclinical drug discovery and development. In: Graham A. Webb, editor. Modern Magnetic Resonance. Dordrecht: Springer 855-877 (2006). 
[18] B.R. Logan, M.P. Geliazkova, D.B. Rowe. An evaluation of spatial thresholding techniques in fMRI analysis. Hum Brain Mapp 29, 1379-1389 (2008).

[19] T. Nichols, A.P. Holmes. Nonparametri permutation tests for functional neuroimaging: a primer with examples. Hum Brain Mapp 15, 1-25 (2001).

[20] T. Nichols, S. Hayasaka. Controlling the familywise error rate in functional neuroimaging: a comparative review. Stat Methods Med Res 12, 419-446 (2003).

[21] P.L. Nunez, R. Srinivasan. Electric fields of the brain. New York: Oxford University Press (2006).

[22] R.D. Pascual-Marqui. Standardized low resolution brain electromagnetic tomography (sLORETA): technical details. Methods Find Exp Clin Pharmacol 24, 5-12 (2002).

[23] K.M. Petersson, T. Nichols, J.B. Poline, A.P. Holmes. Statistical limitations in functional neuroimaging. II. Signal detection and statistical inference. Phil Trans R Soc Lond B 354, 1261-1281 (1999).

[24] J. Riba, P. Anderer, F. Jané, B. Saletu, M.J. Barbanoj. Effects of the south American psychoactive beverage ayahuasca on regional brain electrical activity in humans: a functional neuroimaging study using low-resolution electromagnetic tomography. Neuropsychobiology 40, 89-101 (2004).

[25] S. Romero, M.A. Mañanas, M.J. Barbanoj. A comparative study of automatic techniques for ocular artifact reduction in spontaneous EEG signals based on target variables: a simulation case. Comput Biol Med 38, 348-360 (2008).

[26] S. Romero, M.A. Mañanas, M.J. Barbanoj. Influence of ocular filtering in EEG data on the assessment of drug-induced effects on the brain. Hum Brain Mapp 30, 1470-1480 (2009).

[27] B. Saletu, P. Anderer, G.M. Saletu-Zyhlarz, M. Mandl, O. Arnold, J. Zeitlhofer, et al. EEGtomographic studies with LORETA on vigilance differences between narcolepsy patients and controls and subsequent double-blind, placebo-controlled studies with modafinil. J Neurol 251, 1354-1363 (2004).

[28] B. Saletu, P. Anderer, G.M. Saletu-Zyhlarz. EEG topography and tomography (LORETA) in the classification and evaluation of the pharmacodynamics of psychotropic drugs. Clin EEG Neurosci 37, 66-80 (2006).

[29] B. Saletu, P. Anderer, M. Wolzt, D. Nosiska, A. Assandri, E. Noseda, et al. Double-blind, placebo-controlled, multiple-ascending-dose study on the pharmacodynamics of ABIO$08 / 01$, a new CNS drug with potential anxiolytic activity. Neuropsychobiology 59, 110-122 (2009).

[30] B. Tislerova, M. Brunovsky, J. Horacek, T. Novak, M. Kopecek, P. Mohr, et al. LORETA functional imaging in antipsychotic-naive and olanzapine-, clozapine- and risperidonetreated patients with schizophrenia. Neurophsychobiology 58, 1-10 (2008). 